\title{
Quantum Fisher Information of Decohered W and GHZ Superposition States with Arbitrary Relative Phase
}

\author{
Volkan Erol \\ Okan University Computer Engineering Department Istanbul, 34959 Istanbul, Turkey \\ volkan.erol@gmail.com
}

\begin{abstract}
Quantum Fisher Information (QFI) is a very useful concept for analyzing situations that require phase sensitivity. It become a popular topic especially in Quantum Metrology domain. In this work, we study the changes in quantum Fisher information (QFI) values for one relative arbitrary phased quantum system consisting of a superposition of N Qubits W and GHZ states. In a recent work [7], QFI values of this mentioned system for $\mathrm{N}$ qubits were studied. In this work, we extend this problem for the changes of QFI values in some noisy channels for the studied system. We show the changes in QFI depending on noise parameters. We report interesting results for different type of decoherence channels. We show the general case results for this problem.
\end{abstract}

Keywords: Quantum Fisher Information; arbitrary phase; W State; GHZ State; decoherence

\section{Introduction}

The Quantum Information Theory and Quantum Computation are hot topics that are the theoretical basis of Quantum Computers, which are described as computer technology of the future and intended to operate at very high speeds.

Quantum Fisher Information, a version of Fisher Information, developed for quantum systems, has also become a highly studied subject in recent years, as it also measures the sensitivity that systems can provide for phase sensitive tasks [1-36, 43-44].

Quantum Fisher Information (QFI) is a very useful concept for analyzing situations that require phase sensitivity. This feature has attracted attention and extends the classical Fisher Information. Especially for systems with a higher QFI value, the accuracy is more clearly achieved; For example, clock synchronization [41] and quantum frequency standards [42]. Although some of the pure entangled systems may exceed the classical limit, this does not apply to all entangled systems [37]. The interaction between the quantum system and the environment 
not only reduces entanglement but also reduces the system's Quantum Fisher Information, in general. So we can say that researching quantum systems on QFI is important for the progress of quantum technologies. In recent studies, a single parameter, $\chi^{2}$ parameter, phase sensitivity was added to measure only the self-knowledge of the system under investigation [12]. Since a condition of $\chi^{2}<1$ is not provided for a general quantum system, it is understood that the system has multiple entanglement and this system provides better phase accuracy than a separable system. These quantum systems are called "useful" systems in the literature. For two-level Nparticle quantumsystems, the Cramer-Rao limit is defined by the following formula $[38,39]$ :

$$
\Delta \phi_{Q C B} \equiv \frac{1}{\sqrt{N_{m} F}}
$$

where $N_{m}$ is the number of experiments on the system being measured and $F$ is the Quantum Fisher Information value. We can write 3-dimensional vectors normalized in the nth direction of angular momentum operators, $J_{n}$, Pauli matrices as follows:

$$
J_{\vec{n}}=\sum_{\alpha=x, y, z} \frac{1}{2} n_{\alpha} \sigma_{\alpha}
$$

For $J_{n}$, the Fisher Information of the $\rho$ quantum system can be expressed in a symmetric matrix $C[10]$ :

$$
F\left(\rho, J_{\vec{n}}\right)=\sum_{i \neq j} \frac{2\left(p_{i}-p_{j}\right)^{2}}{p_{i}+p_{j}}\left|\left\langle i\left|J_{\vec{n}}\right| j\right\rangle\right|^{2}=\vec{n} C \vec{n}^{T}
$$

where $p_{i}$ and $|i\rangle$ represent the eigenvalues and eigenvectors of the $\rho$ system, respectively, and the matrix $C$ is defined as

$$
C_{k l}=\sum_{i \neq j} \frac{\left(p_{i}-p_{j}\right)^{2}}{p_{i}+p_{j}}\left[\left\langle i\left|J_{k}\right| j\right\rangle\left\langle j\left|J_{i}\right| i\right\rangle+\left\langle i\left|J_{i}\right| j\right\rangle\left\langle j\left|J_{k}\right| i\right\rangle\right]
$$

The largest $F$ value between the $N$ options is selected and averaged over $N$ particles. The Fisher Information value is calculated as the greatest eigenvalue of the $C$ matrix. This definition is expressed by the equation: 


$$
\bar{F}_{\max }=\frac{1}{N} \max _{\vec{n}} F\left(\rho, J_{\vec{n}}\right)=\frac{\lambda_{\max }}{N}
$$

\section{Quantum State with Arbitrary Relative Phase and Reported Results}

In this section, we introduce $\mathrm{N}$ qubit systems in decoherence channels. Particularly the system studied is superposition of a $\mathrm{W}$ and a GHZ state [7] and,

$$
\left|\varphi^{N}\right\rangle=\alpha e^{i \mu}\left|G H Z^{N}\right\rangle+\beta e^{i v}\left|W^{N}\right\rangle+\gamma\left|\bar{W}^{N}\right\rangle
$$

Here $N$ is the number of qubits, and $\alpha, \beta$ and $\gamma$ are the superposition coefficient. In this work, we consider the case for $N=3,4$ and 5 . Here $\boldsymbol{\beta}=\sqrt{\mathbf{1}-\left(\boldsymbol{\alpha}^{2}+\boldsymbol{\gamma}^{\mathbf{2}}\right)}$ and $\overline{\boldsymbol{W}}$ is the $\mathrm{W}$ state in which Pauli spin matrix $\left(\alpha_{X}\right)$ is applied to each qubit. The decoherence channels are amplitude damping (ADC), phase damping (PDC) and depolarizing (DPC) respectively. Then we study the QFI of the $\mathrm{N}$ qubit systems for di erent scenarios. As a first scenario, we take the superposition coeffients $\alpha=0.6$ and relative phase parameters $\mu=\pi$ and $v=-\pi / 9$ and $\gamma$ is variable in $(0,0.8)$ interval and we apply ADC to systems $\left|\boldsymbol{\varphi}^{3}\right\rangle,\left|\boldsymbol{\varphi}^{\mathbf{4}}\right\rangle$ and $\left|\boldsymbol{\varphi}^{5}\right\rangle$. In Figure 1, changes in QFI values per particle for ADC are showed.

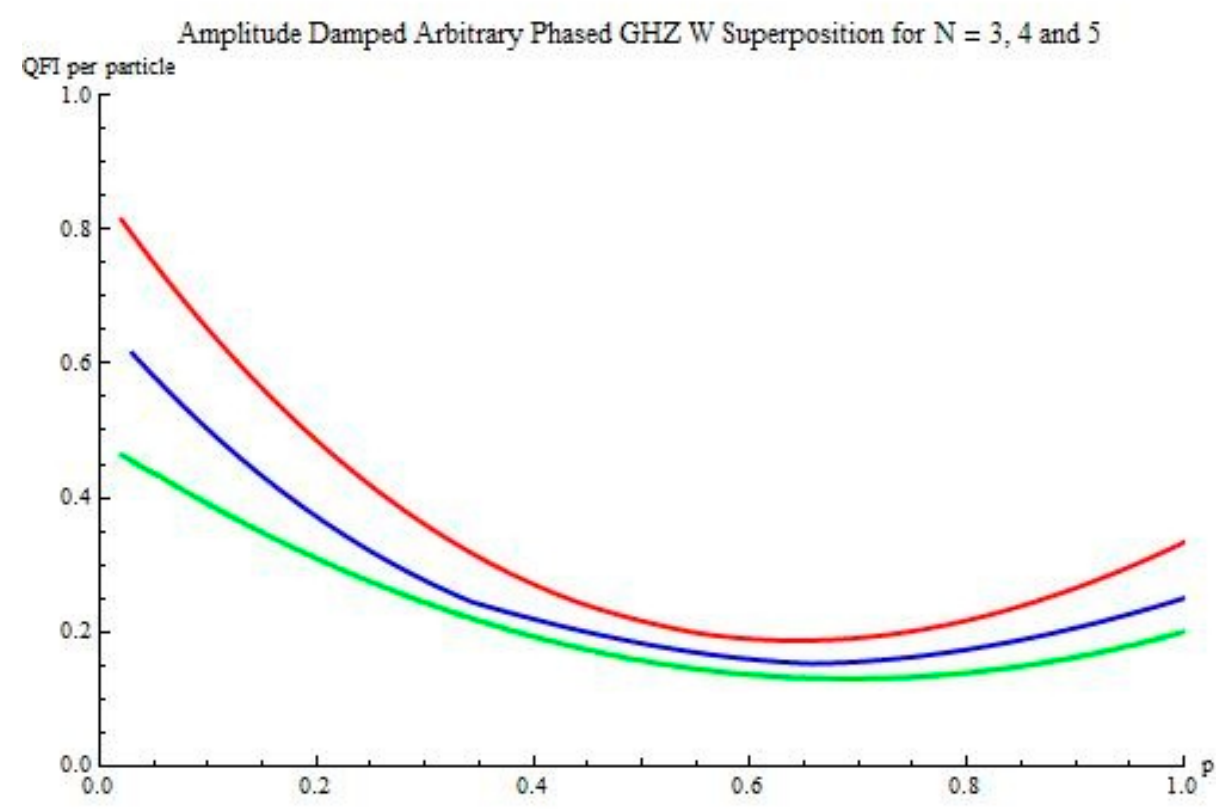

Fig. 1. Changes in QFI per particle for $A D C$ with changing $p$ values (Red: $N=3$, Blue: $N=4$, Green: $N=5$ )

In the second scenario, we apply PDC the same system with same values. In Figure 2, changes in QFI values per particle for PDC are shown. 
Phase Damped Arbitrary Phased GHZ W Superposition for $\mathrm{N}=3,4$ and 5

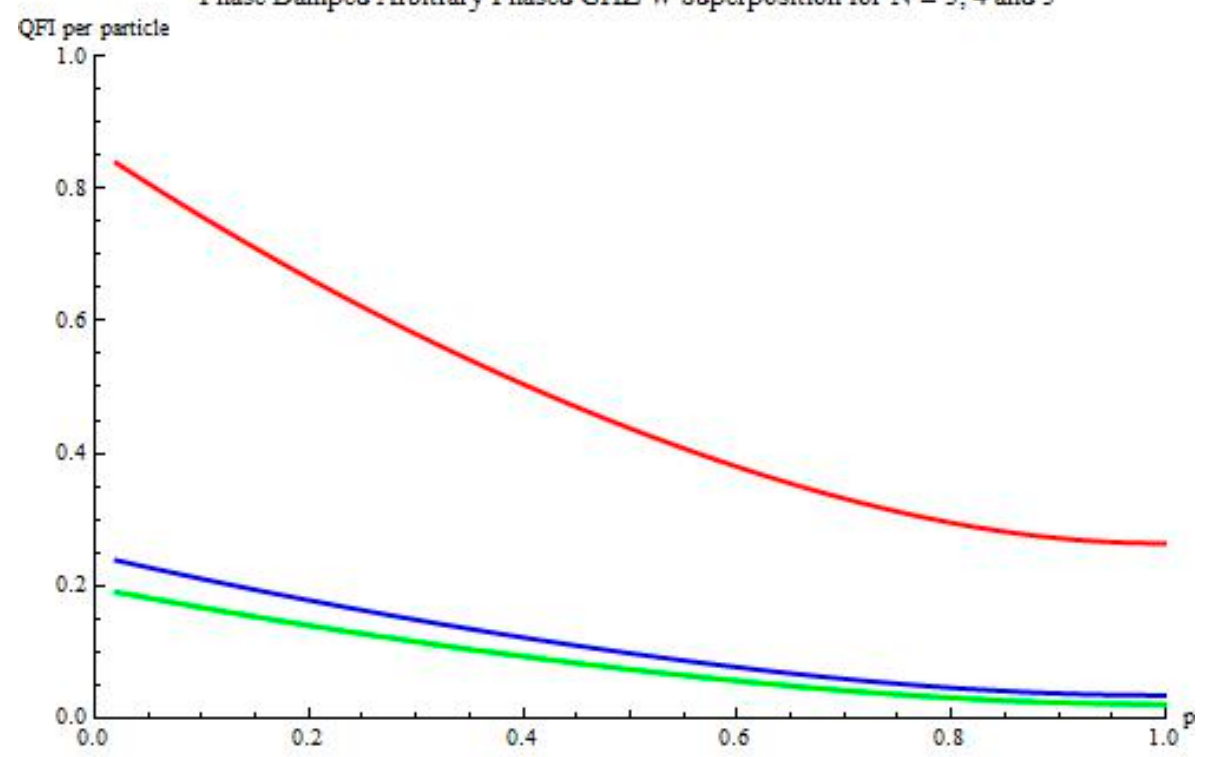

Fig. 2. Changes in QFI per particle for PDC with changing $p$ values (Red: $N=3$, Blue: $N=4$, Green: $N=5$ )

In the third scenario, we apply PDC the same system with same values. In Figure 2, changes in QFI values per particle for PDC are shown.

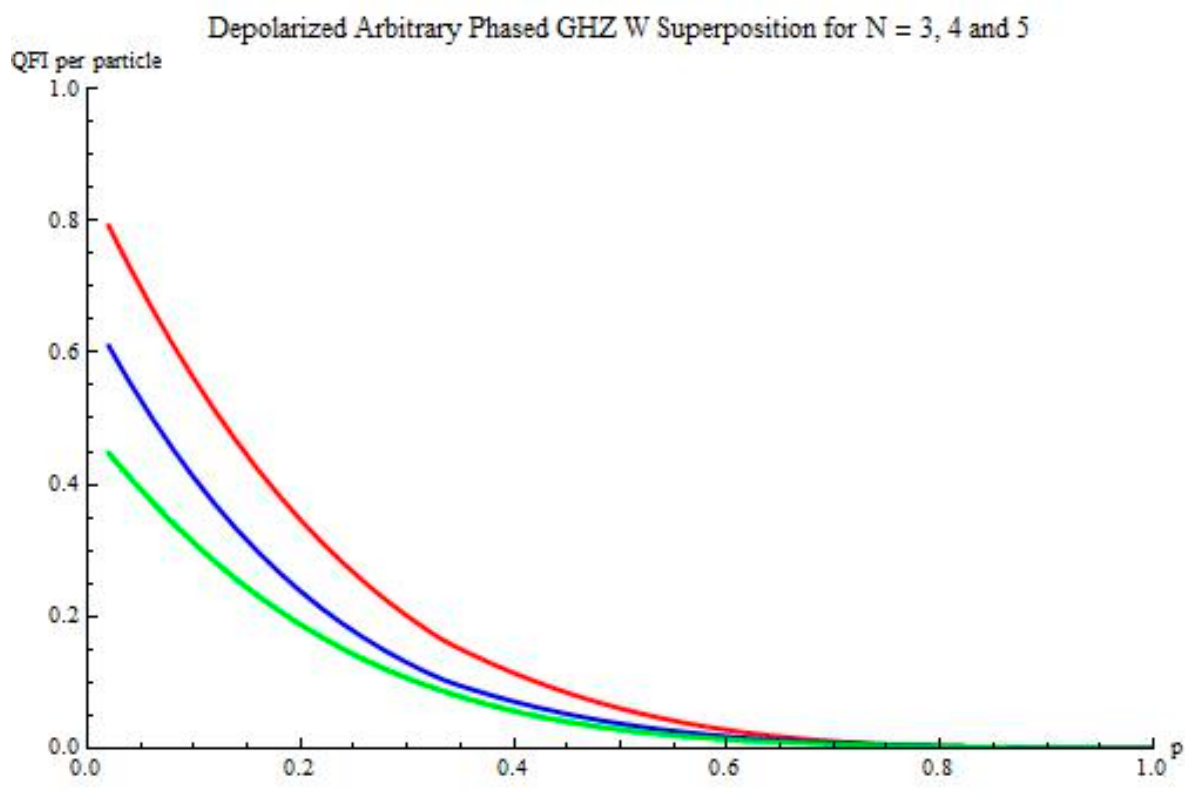

Fig. 3. Changes in QFI per particle for DPC with changing $p$ values (Red: $N=3$, Blue: $N=4$, Green: $N=5$ )

In APC scenario, we see minimum points for all $\mathrm{N}$ values for value of $\mathrm{p}=\sim 0.65$. Until this point QFI per particle decreases and after it increases. For all $\mathrm{N}$ values, even if there is a minimum 
QFI per particle remains greater than $\sim 0,15$ value. We can say that this quantum system is more resistant to APC noise than the others.

In PDC scenario, the QFI per particle values are always decreasing, the minimum is for $\mathrm{p}=1$ for each value of $\mathrm{N}$. For the system $\mathrm{N}=3$, it is significantly more resistant to PDC noise than the systems for values $\mathrm{N}=4$ and $\mathrm{N}=5$.

In DPC scenario, the QFI per particle values are always decreasing and surprisingly DPC destroys completely QFI after values $\mathrm{p}=\sim 0.8$ for each value of $\mathrm{N}$.

In the fourth scenario, we find the QFI per particle as functions of $\gamma$ and $p$. Surprisingly the QFI per particle values remains the same when $\gamma$ values change. This is an important result observed during this study.

In Figure 4, we show in 3D plot, the changes of QFI per particle values under each decoherence channel for values $\mathrm{N}=3, \mathrm{~N}=4$ and $\mathrm{N}=5$, respectively. 

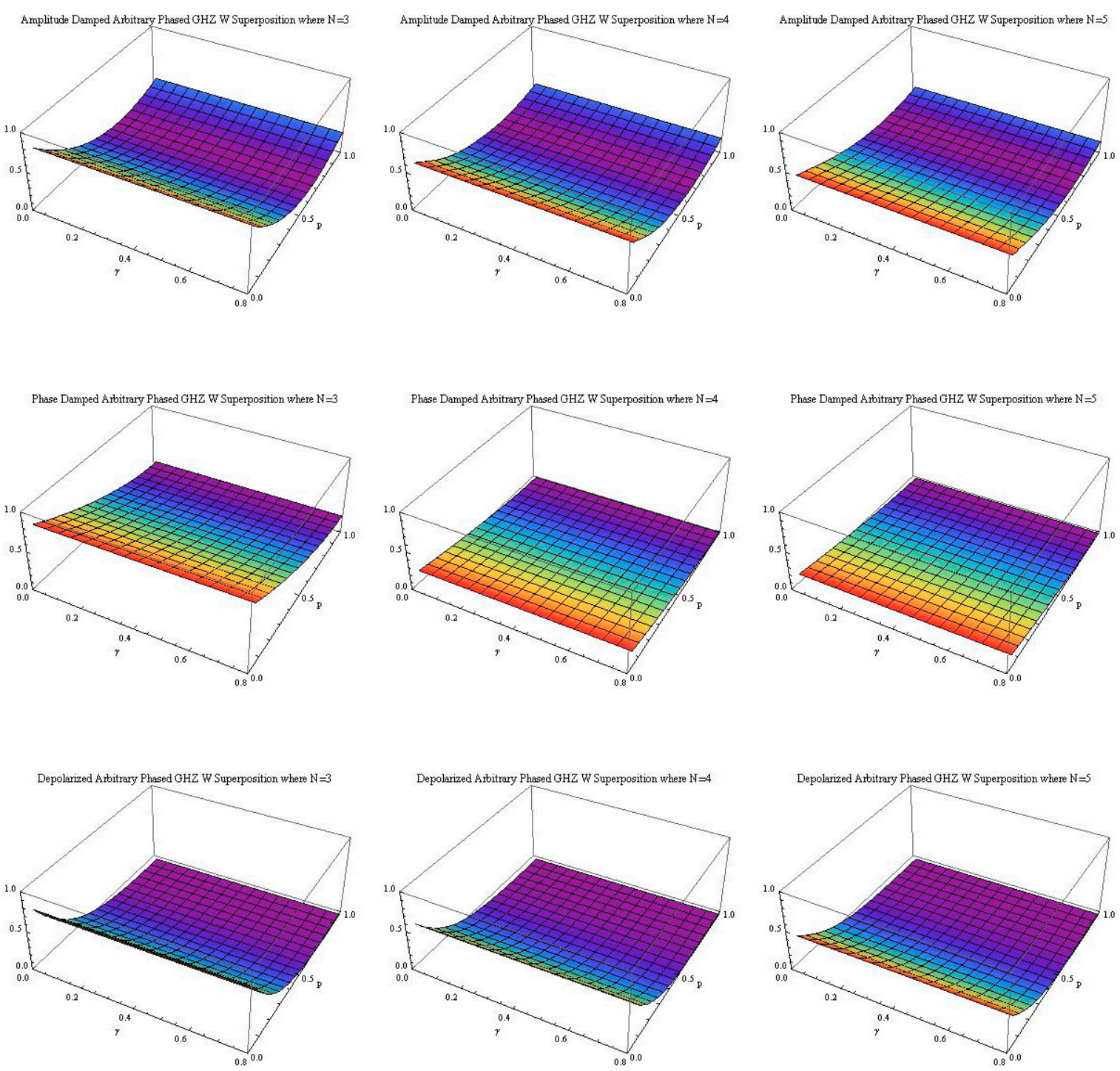

Fig. 4. QFI values as function of $\gamma$ and $\mathrm{p}$ (3D plot)

\section{Conclusion}

We studied the changes in QFI values for a useful quantum system state which is a superposition of $\mathrm{W}_{\mathrm{N}}$ and $\mathrm{GHZ}_{\mathrm{N}}$ states where $\mathrm{N}=3,4$ and 5. In this work, we extended this problem for the mentioned arbitrary phased system and we observed the changes of QFI values/per particle in amplitude damping, phase damping and depolarizing channels. We showed the changes in QFI depending on noise parameters and superposition coefficient. Surprisingly the QFI per particle values remains the same when $\gamma$ values change. DPC destroys completely QFI after values 
$\mathrm{p}=\sim 0.8$ for each value of $\mathrm{N}$. And studied quantum system is more resistant to APC noise than the others.

\section{References}

1. V. Erol, Quantum Fisher Information of W and GHZ State Superposition under Decoherence. Preprints 2017, 2017040147 (doi: 10.20944/preprints201704.0147.v1), 2017.

2. Z. Ji et. al, Parameter Estimation of Quantum Channels, IEEE Trans. Info. Theory., vol. 54, no. 5172, 2008.

3. B. M. Escher, M. Filho, and L. Davidovich, General framework for estimating the ultimate precision limit in noisy quantum-enhanced metrology, Nat. Phys., vol. 7, no. 406, 2011.

4. X. Yi., G. Huang and J. Wang, Quantum Fisher Information of a 3-Qubit State, Int. J. Theor. Phys. vol. 51, p. 3458, 2012.

5. N. Spagnolo et al., Quantum interferometry with three-dimensional geometry, Nature Sci. Rep., vol. 2, no. 862, 2010.

6. Z. Liu, Spin Squeezing in Superposition of Four-Kübit Symmetric State and W States, Int. J. Theor. Phys., vol. 52, p. 820, 2013.

7. F. Ozaydin, A. A. Altintas, S. Bugu and C. Yesilyurt, Quantum Fisher Information of N Particles in the Superposition of W and GHZ States, Int. J. Theor. Phys., vol. 52, p. 2977, 2013.

8. F. Ozaydin, A. A. Altintas, S. Bugu, C. Yesilyurt and M.Arik, Quantum Fisher Information of Several Qubits in the Superposition of A GHZ and two W States with Arbitrary Relative Phase, Int. J. Theor. Phys., vol. 53, p. 3259, 2014.

9. P. Gibilisco, D. Imparato, and T. Isola, Uncertainty principle and Quantum Fisher Information, J. Math. Phys., vol. 48, no. 072109, 2007.

10. A. Andai, Uncertainty principle with Quantum Fisher Information, J. Math. Phys., vol. 49, no. $012106,2008$.

11. J. Ma, Y. Huang, X. Wang and C. P. Sun, Quantum Fisher information of the GreenbergerHorne-Zeilinger state in decoherence channels, Phys. Rev. A, vol. 84, no. 022302, 2011.

12. Toth et al., Spin squeezing and entanglement, Phys. Rev. A, vol. 79, no. 042334, 2009.

13. F.Ozaydin, A.A.Altintas, S.Bugu, C.Yesilyurt, Behavior of Quantum Fisher Information of Bell Pairs under Decoherence Channels, Acta Phys. Pol. A, vol. 125(2), p. 606, 2014.

14. S.Z.Ang, G.I.Harris, W.P.Bowen and M.Tsang, Optomechanical parameter estimation”, New J. Phys., vol. 15, no. 103028, 2013.

15. K.Iwasawa et al., Quantum-Limited Mirror-Motion Estimation, Phys. Rev. Lett., vol. 111, no. $163602,2013$. 
16. M.Tsang, Quantum metrology with open dynamical systems, New J. Phys., no. 15, vol. 073005, 2013.

17. M.Tsang and N.Ranjith, Fundamental quantum limits to waveform detection, Phys. Rev. A, vol. 86 , no. $042115,2012$.

18. M.Tsang, Ziv-Zakai Error Bounds for Quantum Parameter Estimation, Phys. Rev. Lett., vol. 108, no. 230401, 2012.

19. M.Tsang. H.M.Wiseman, C.M.Caves, Fundamental Quantum Limit to Waveform Estimation, IEEE Conference on Lasers and Electro-Optics (CLEO) 2011.

20. M.Tsang, J.H.Shapiro, S.Lloyd, Quantum Optical Temporal Phase Estimation by Homodyne Phase-Locked Loops, IEEE Conference on Lasers and Electro-Optics (CLEO) 2009.

21. F. Ozaydin, Phase damping destroys quantum Fisher information of W states, Phys. Let. A, vol. 378, pp. 3161-3164, 2014.

22. V. Erol, F. Ozaydin, A. A. Altintas, Analysis of entanglement measures and locc maximized quantum fisher information of general two qubit systems, Sci. Rep. 4, 5422, 2014.

23. F. Ozaydin, A. A. Altintas, C. Yesilyurt, S. Bugu, V. Erol, Quantum Fisher Information of Bipartitions of W States, Acta Physica Polonica A 127, 1233-1235, 2015.

24. V. Erol, S. Bugu, F. Ozaydin, A. A. Altintas, An analysis of concurrence entanglement measure and quantum fisher information of quantum communication networks of two-qubits, Proceedings of IEEE 22nd Signal Processing and Communications Applications Conference (SIU2014), pp. 317-320, 2014.

25. V. Erol, A comparative study of concurrence and negativity of general three-level quantum systems of two particles, AIP Conf. Proc. 1653 (020037), 2015.

26. V. Erol, F. Ozaydin, A. A. Altintas, Analysis of Negativity and Relative Entropy of Entanglement measures for qubit-qutrit Quantum Communication systems, Proceedings of IEEE 23rd Signal Processing and Communications Applications Conference (SIU2015), pp. 116-119, 2014.

27. V. Erol, Detecting Violation of Bell Inequalities using LOCC Maximized Quantum Fisher Information and Entanglement Measures, Preprints 2017, 2017030223 (doi: 10.20944/preprints201703.0223.v1), 2017.

28. V. Erol, Analysis of Negativity and Relative Entropy of Entanglement Measures for Two Qutrit Quantum Communication Systems, Preprints 2017, 2017030217 (doi: 10.20944/preprints201703.0217.v1), 2017.

29. V. Erol, The relation between majorization theory and quantum information from entanglement monotones perspective, AIP Conf. Proc. 1727 (020007), 2016. 
30. V. Erol, A Proposal for Quantum Fisher Information Optimization and its Relation with Entanglement Measures, PhD Thesis, Okan University, Institute of Science, 2015.

31. F. Ozaydin, A. A. Altintas, Quantum Metrology: Surpassing the shot-noise limit with Dzyaloshinskii-Moriya interaction, Sci. Rep. 5, 16360, 2015.

32. A. A. Altintas, Quantum Fisher Information of an open and noisy system in the steady state, Annals of Physics, 362, pp: 192-198, 2016.

33. F. Ozaydin, Quantum Fisher Information of a $3 \times 3$ Bound Entangled State and its Relation with Geometric Discord, International Journal of Theoretical Physics 54 (9), 3304-3310, 2015.

34. B. R. Frieden and R. A. Gatenby, Exploratory Data Analysis Using Fisher Information, Arizona, USA:Springer, 2007.

35. L. Pezze, A. Smerzi, Entanglement, Nonlinear Dynamics, and the Heisenberg Limit, Phys. Rev. Lett., vol. 102, no. 100401, 2009.

36. V. Giovannetti, S. Lloyd and L. Maccone, Quantum-Enhanced Measurements: Beating the Standard Quantum Limit, Science, vol. 306, no. 1330, 2004.

37. P. Hyllus, O. Gühne and A. Smerzi, Not all pure entangled states are useful for sub-shotnoise interferometry, Phys. Rev. A, vol. 82, no. 012337, 2010.

38. C. W. Helstrom, Quantum Detection and Estimation Theory, New York: Academic Press, 1976.

39. A. S. Holevo, Probabilistic and Statistical Aspects of Quantum Theory, Amsterdam, The Netherlands: North-Holland, 1982.

40. H. N. Xiong, J. Ma, W. F. Liu and X. Wang, Quantum Fisher Information for Superpositions of Spin States, Quant. Inf. Comp., vol.10 no.5\&6, 2010.

41. R. Jozsa, D. S. Abrams, J. P. Dowling, and C. P. Williams, Quantum Clock Synchronization Based on Shared Prior Entanglement, Phys. Rev. Lett., vol. 85, no. 2010, 2000.

42. J. J. Bollinger, W. M. Itano, D. J. Wineland, and D. J. Heinzen, Optimal frequency measurements with maximally correlated states, Phys. Rev. A, vol. 54, no.R 4649, 1996.

43. V. Erol, Quantum Fisher Information: Theory and Applications. Preprints 2017, 2017040134 (doi: 10.20944/preprints201704.0134.v1), 2017.

44. V. Erol, Entanglement Monotones and Measures: An Overview. Preprints 2017, 2017040098 (doi: 10.20944/preprints201704.0098.v3), 2017. 